University of Nebraska - Lincoln

DigitalCommons@University of Nebraska - Lincoln

Timothy J. Gay Publications

Research Papers in Physics and Astronomy

August 1986

New insights into Mott-scattering electron polarimetry

G. D. Fletcher

Yale University, New Haven, Connecticut

Timothy J. Gay

University of Nebraska - Lincoln, tgay1@unl.edu

M. S. Lubell

City College of the City University of New York, New York, New York

Follow this and additional works at: https://digitalcommons.unl.edu/physicsgay

Part of the Physics Commons

Fletcher, G. D.; Gay, Timothy J. ; and Lubell, M. S., "New insights into Mott-scattering electron polarimetry" (1986). Timothy J. Gay Publications. 13.

https://digitalcommons.unl.edu/physicsgay/13

This Article is brought to you for free and open access by the Research Papers in Physics and Astronomy at DigitalCommons@University of Nebraska - Lincoln. It has been accepted for inclusion in Timothy J. Gay Publications by an authorized administrator of DigitalCommons@University of Nebraska - Lincoln. 
Phys. Rev. A 34, 911 - 923 (1986)

[Issue 2 - August 1986]

\title{
New insights into Mott-scattering electron polarimetry
}

\author{
G. D. Fletcher \\ Department of Physics, Yale University, New Haven, Connecticut 06520 and \\ Department of Physics, University of Virginia, Charlottesville, Virginia 22901
}

T. J. Gay

Department of Physics, Yale University, New Haven, Connecticut 06520 and

Department of Physics, University of MissouriRolla, Rolla, Missouri 65401

M. S. Lubell

Department of Physics, City College of the City University of New York, New York, New York 10031

\section{Received 5 February 1986}

In order to understand some of the systematic effects associated with conventional Mottscattering electron polarization measurements, we analyzed asymmetry data obtained with 94-keV polarized electrons scattered at $120^{\circ}$ from gold-foil targets ranging in thickness from 27 to $62 \mu \mathrm{g} / \mathrm{cm}^{2}$. Based upon an examination of the influence of multiple and plural scattering, we conclude that the precision of such Mott measurements is fundamentally limited by an experimental uncertainty of greater than $\pm 2.5 \%$ and an absolute uncertainty of approximately $\pm 5 \%$, considerably worse than is customarily assumed. We believe that similar conclusions can be drawn for measurements made with "compact" cylindrical and spherical Mott polarimeters.

C1986 The American Physical Society

URL: http://link.aps.org/abstract/PRA/v34/p911

DOI: 10.1103/PhysRevA.34.911

PACS: $34.80 . \mathrm{Nz}$ 


\title{
New insights into Mott-scattering electron polarimetry
}

\author{
G. D. Fletcher* \\ Department of Physics, Yale University, New Haven, Connecticut 06520 \\ and Department of Physics, University of Virginia, Charlottesville, Virginia 22901 \\ T. J. Gay ${ }^{\dagger}$ \\ Department of Physics, Yale University, New Haven, Connecticut 06520 \\ and Department of Physics, University of Missouri-Rolla, Rolla, Missouri 65401 \\ M. S. Lubell \\ Department of Physics, City College of the City University of New York, New York, New York 10031 \\ (Received 5 February 1986)

\begin{abstract}
In order to understand some of the systematic effects associated with conventional Mottscattering electron polarization measurements, we analyzed asymmetry data obtained with 94-keV polarized electrons scattered at $120^{\circ}$ from gold-foil targets ranging in thickness from 27 to 62 $\mu \mathrm{g} / \mathrm{cm}^{2}$. Based upon an examination of the influence of multiple and plural scattering, we conclude that the precision of such Mott measurements is fundamentally limited by an experimental uncertainty of greater than $\pm 2.5 \%$ and an absolute uncertainty of approximately $\pm 5 \%$, considerably worse than is customarily assumed. We believe that similar conclusions can be drawn for measurements made with "compact" cylindrical and spherical Mott polarimeters.
\end{abstract}

\section{INTRODUCTION}

During the last twenty years, polarized electron sources have undergone substantial development, ${ }^{1-7}$ resulting in the increased application of polarized electron beams to a variety of experimental studies. Their usage received a major impetus in 1975 with the first report of the GaAs photoemission source. ${ }^{8}$ Although limited by a valenceband degeneracy to a polarization of $\leq 0.5$, the GaAs source produces extremely high electron currents ${ }^{9}$ of intrinsically high brightness and narrow energy spread, ${ }^{10,11}$ characteristics which make it the undisputed choice for most polarized electron applications.

The growth of the polarized electron "industry," not unexpectedly, has created an increasing need for precise, reliable electron polarimeters. To date, despite occasional uses of other polarimeters, high-energy $(30-120 \mathrm{keV})$ Mott scattering ${ }^{1,5,6,12-21}$ still serves as the industry standard, ${ }^{22-36}$ as it has since the late 1950 's. ${ }^{37,38}$ It is pertinent, therefore, to address the issue of the limitations of Mott-scattering measurements as reflected by systematic uncertainties that affect the absolute determinations of electron polarization.

Until fairly recently, Mott polarimeters tended to be of a standard design ${ }^{22}$ in which transversely polarized electrons are first accelerated to energies of approximately $100 \mathrm{keV}$ in a conventional accelerating column and subsequently allowed to strike thin gold-foil targets. Counting rates for electrons elastically scattered at $120^{\circ}$ in a plane perpendicular to their polarization vector $\mathbf{P}$ are then measured by energy-sensitive detectors arranged $180^{\circ}$ apart in azimuthal angle. Figures 1 and 2 illustrate respectively the typical layout and scattering geometry of such a polarimeter. $^{22}$
As is evident from Fig. 1, conventional Mott scattering devices are rather cumbersome in part because their highvoltage accelerating column is exposed and in part because their detectors must be maintained at high voltage. Moreover they suffer from poor discrimination against inelastically scattered electrons, a consequence of the intrinsic resolution of most energy-sensitive detectors. In light of these liabilities, the conventional Mott polarimeter has been increasingly replaced by more compact devices $^{25,32,33}$ in which scattering energies are reduced to less than $50 \mathrm{keV}$, a single-stage internal accelerating geometry is used instead of the exposed column, and channel electron multipliers biased close to ground potential are employed in place of energy-sensitive detectors floated at high voltage. The cylindrical or spherical geometries of the compact polarimeters, shown in Figs. 3(a) and 3(b), respectively, allow inelastic scattering events to be eliminated electrostatically with a resolution much higher than that obtainable with energy-sensitive detectors. While the compact Mott devices greatly improve the ease with which electron polarizations can be measured, they still suffer from a number of the same fundamental limitations of conventional Mott polarimeters. In this paper we discuss these limitations based upon measurements made using a conventional polarimeter.

The underlying physics of Mott scattering, or more precisely "high-energy" Mott scattering, ${ }^{6}$ is the spin-orbit interaction, which is enhanced by the use of high- $Z$ targets, typically thin gold foils. The generalized differential elastic Mott cross section can be written as ${ }^{1}$

$$
\frac{d \sigma(\theta)}{d \theta}=I(\theta)[1+S(\theta) \mathbf{P} \cdot \hat{\mathbf{n}}],
$$

where $\mathbf{P}$ is the polarization vector of the incident elec- 


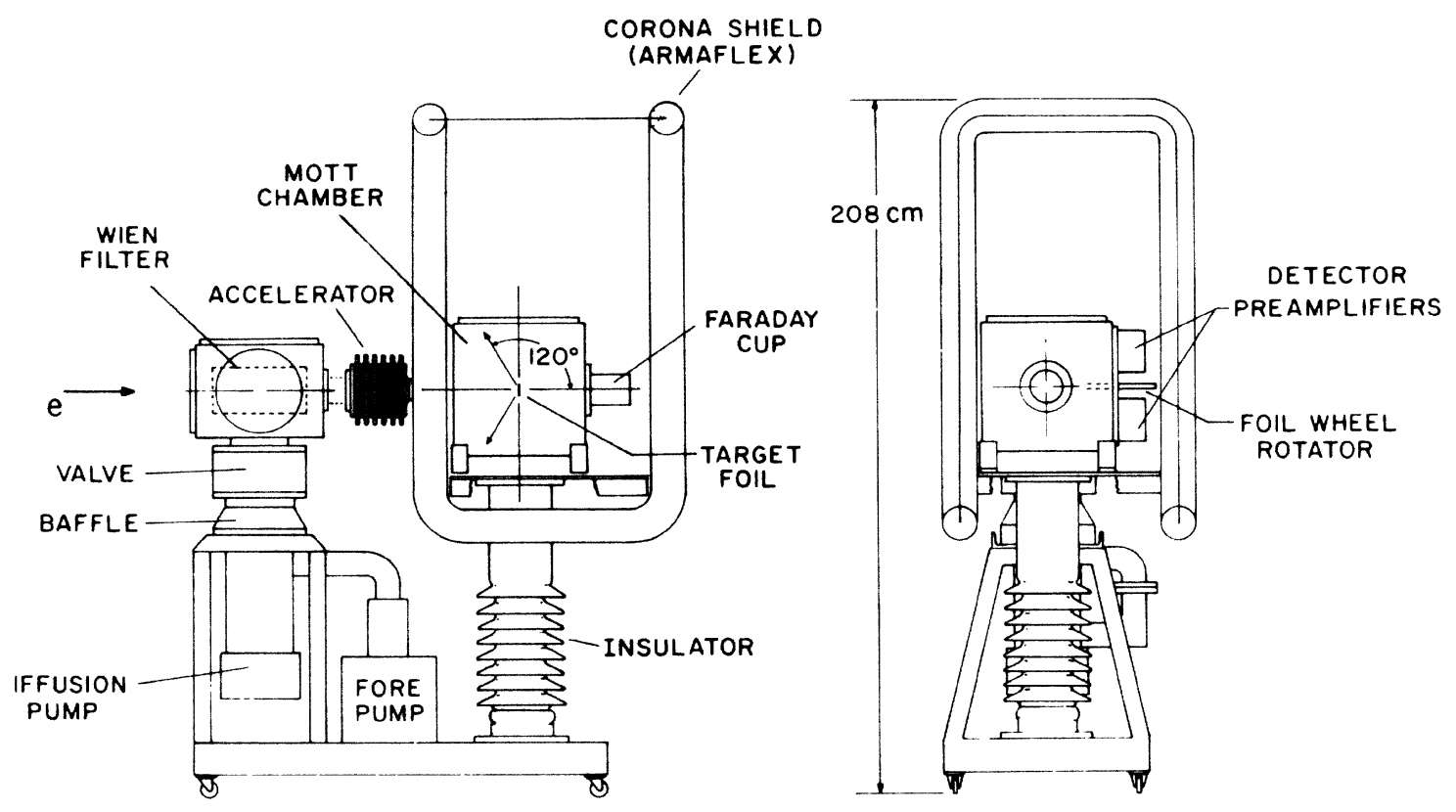

FIG. 1. Scale drawing of standard Mott-scattering electron polarimeter (Ref. 22) (side and end views). The Wien-filter spin rotator consists of crossed transverse electric and magnetic fields of $1.91 \mathrm{kV} / \mathrm{cm}$ and $39 \mathrm{G}$, respectively. With the Wien filter maintained at $+6 \mathrm{kV}$ with respect to ground, the beam energy in the Wien filter is $7 \mathrm{keV}$. The accelerator tube consists of seven aluminum disks connected by $1.7-\mathrm{G} \Omega$ resistors. The entire Mott-scattering region, including detectors, preamplifiers, amplifiers, and detector bias supplies is maintained at a potential of $+93 \mathrm{kV}$, providing a beam energy of $94 \mathrm{keV}$.

trons, $\hat{\mathbf{n}}$ is the unit vector normal to the scattering plane shown in Fig. 4, and $I(\theta)$ and $S(\theta)$ are defined by

$$
I(\theta)=|F(\theta)|^{2}+|G(\theta)|^{2}
$$

and

$$
S(\theta)=i \frac{F(\theta) G^{*}(\theta)-F^{*}(\theta) G(\theta)}{I(\theta)}
$$

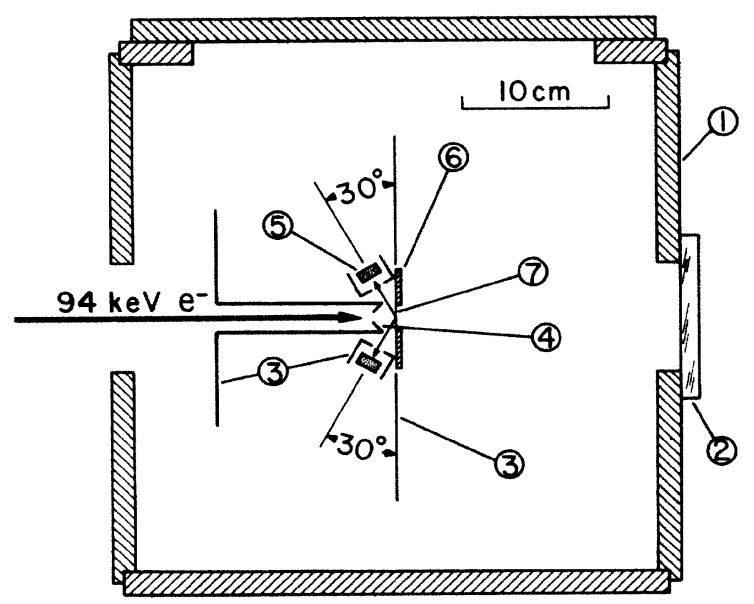

FIG. 2. Geometry of Mott scattering region. The 94-keV transversely polarized electrons enter from the left and are scattered by one of four gold targets in the target wheel, which can be rotated while the system is under vacuum and at high voltage. The following elements are indicated: (1) aluminum vacuum chamber, (2) Lucite window, (3) aluminum shielding, (4) aluminum beam collimator, (5) surface-barrier detector, (6) target wheel, (7) gold-foil target. with $F(\theta)$ and $G(\theta)$ respectively denoting the spinnonflip and spin-flip scattering amplitudes. Provided $\mathbf{P}$ is not purely longitudinal, it is clear from Eq. (1) that the differential cross section depends not only on $S(\theta)$ but also on the azimuthal angle $\phi$. The latter dependence enters through the definition of $\hat{\mathbf{n}}$ :

$$
\hat{\mathbf{n}} \equiv \hat{\mathbf{k}}_{1} \times \hat{\mathbf{k}}_{2},
$$

where $\mathbf{k}_{1}$ is the momentum of the incident electron and $\mathbf{k}_{2}$ the momentum of the scattered electron. If, for example, two ideal detectors are located $\pi$ radians $\left(180^{\circ}\right)$ apart in azimuth and $\mathbf{P}$ is oriented perpendicular to the scattering plane, an ideal Mott asymmetry $\delta_{M}(\theta)$ can be defined by

$$
\delta_{M}(\theta)=\frac{N_{2}(\theta)-N_{1}(\theta)}{N_{2}(\theta)+N_{1}(\theta)},
$$

where $N_{2}(\theta)=N(\theta,+\pi / 2)$ and $N_{1}(\theta)=N(\theta,-\pi / 2)$ are the counting rates of the two detectors. With the use of Eq. (1), $\delta_{M}(\theta)$ in turn can be expressed as

$$
\delta_{M}(\theta)=P S(\theta) .
$$

The sensitivity of the polarimeter thus depends directly on $S(\theta)$, as a consequence of which this function is customarily called the "analyzing power." Although many calculations of $S(\theta)$ have been performed through the years, ${ }^{12,39-47}$ the first accurate calculations incorporating screening were carried out by Sherman, for gold $(Z=79)$, cadmium $(Z=48)$, and aluminum $(Z=13))^{48,49}$ Consequently the Mott analyzing power is also known as the "Sherman function."

While the fundamental physics of Mott scattering, as described by Eqs. (1)-(6), is well understood, the com- 
plexities introduced by any practical apparatus pose problems that make the interpretation of polarization measurements somewhat difficult. In the following sections we will examine some of the systematic effects that complicate such measurements and investigate the limits that
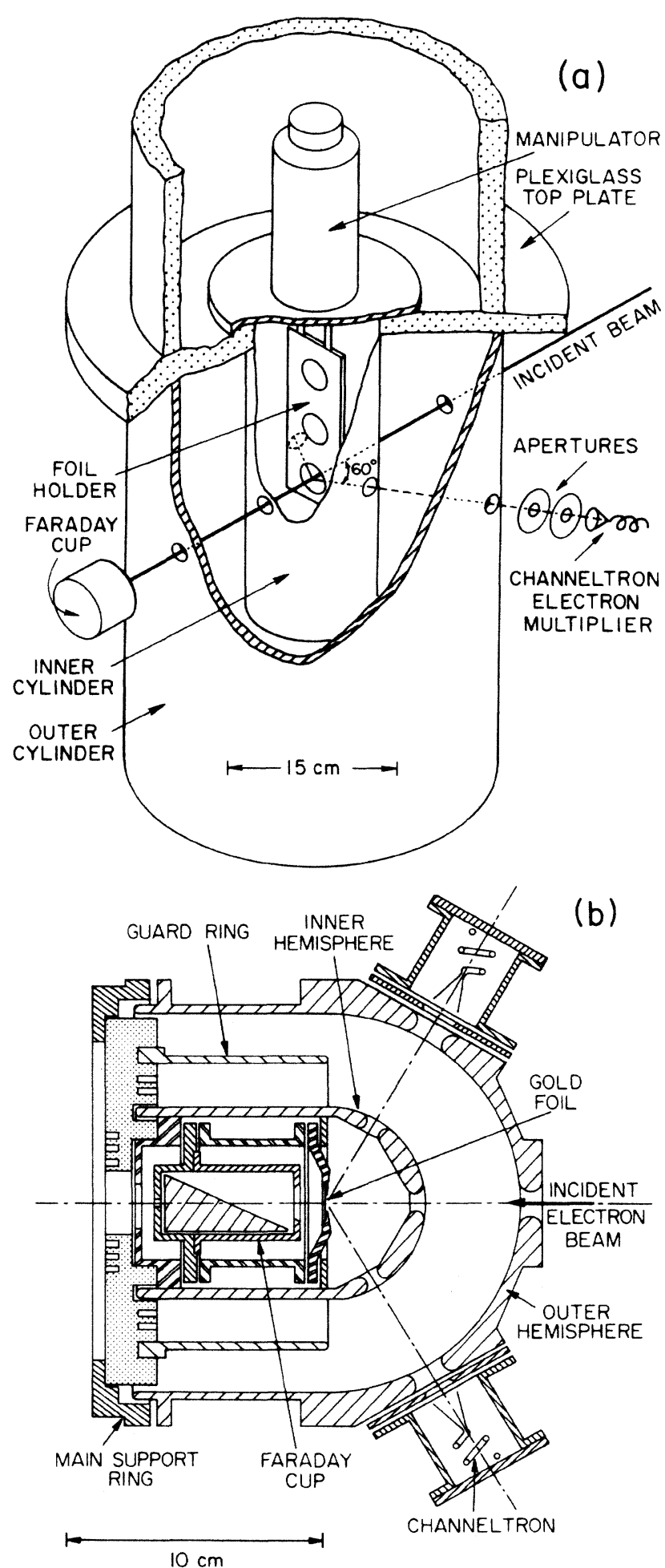

FIG. 3. Schematic diagram of essential components of (a) cylindrical Mott polarimeter taken from Ref. 25 and (b) spherical Mott polarimeter taken from Ref. 32.

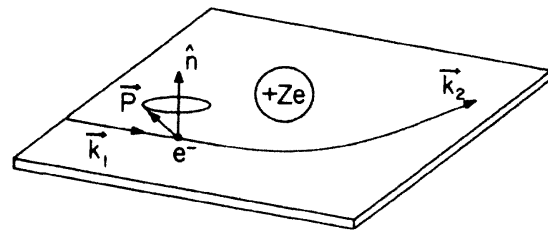

FIG. 4. Kinematics of Mott scattering showing initial and final momentum vectors $\mathbf{k}_{1}$ and $\mathbf{k}_{2}$, respectively, polarization vector $\mathbf{P}$, and unit vector $\hat{\mathbf{n}}$ normal to the scattering plane.

they place on the ultimate precision of Mott polarimetry. We will also address the question of future Mott scattering needs in light of our conclusions.

\section{EXPERIMENTAL APPARATUS AND DATA ACQUISITION}

The polarized electron apparatus used in our Mott scattering studies has been described in detail elsewhere, ${ }^{22,34,50-53}$ with scale drawings of the various components appearing in Refs. 22 and 34 . For clarity, we now summarize the relevant features of the experimental configuration.

Longitudinally polarized $1-\mathrm{keV}$ electrons emerging from a Fano-effect source ${ }^{22}$ were deflected by a $45^{\circ}$ bending magnet into the polarimeter beam line and transported by a series of magnetic lenses and steering coils to a Wien-filter ${ }^{54}$ spin-rotator unit. In this unit, the beam was first accelerated to $7 \mathrm{keV}$ (to minimize the effects of fringing fields) and then focused by an electrostatic quadrupole doublet into the Wien-filter proper, which had an effective length of $11.7 \mathrm{~cm}$ and a field plate separation of 1.27 $\mathrm{cm}$ and which employed crossed transverse electric and magnetic fields of $1.9 \mathrm{kV} / \mathrm{cm}$ and $39 \mathrm{G}$, respectively. The transversely polarized electrons emerging from the spin rotator were then transported through an electrostatic steering section and accelerated to approximately $94 \mathrm{keV}$ $(\beta=0.53)$ in a conventional high-voltage column preparatory to Mott-scattering analysis.

Upon entering the Mott chamber, the "high-energy" electrons, with their polarization vector oriented horizontally, passed through an aluminum shield and collimation assembly, which served both to define the beam and shield the detectors from stray electrons, as suggested by the geometry shown in Fig. 2. Electrons scattered through $120^{\circ}$ by gold-foil targets located downstream from the end of the tube were detected by two silicon surface barrier detectors (Ortec SBEE 100) situated $180^{\circ}$ apart azimuthally in the vertical plane. Each detector subtended a solid angle of $0.14 \mathrm{sr}$, defined by an entrance aperture in its aluminum housing.

The target foils, $1.5 \mathrm{~cm}$ in diameter, were mounted on an aluminum wheel that could be rotated under vacuum. In the study covered by this report, four gold foils were used with areal densities of $27,44,53$, and $62 \mu \mathrm{g} / \mathrm{cm}^{2}$. The foils were prepared by vacuum deposition on "lowmolecular-weight" Formvar (Monsanto type 7/95E) backings approximately $20 \mu \mathrm{g} / \mathrm{cm}^{2}$ in thickness. In addition to the four gold targets, the target wheel contained a bare 
Formvar foil and a ${ }^{109} \mathrm{Cd}$ internal-conversion electron source for energy calibration, the latter deposited on a thin nickel foil. The thicknesses of the gold foils were measured relatively to accuracies of $\pm 5 \%$ by a piezoelectric frequency monitor (Sloan Instruments model DTM-3) used during the vacuum deposition process. In order to place the thickness on an absolute scale, interferometric measurements were performed on a glass flat that had been exposed during the deposition process. While not particularly germane to the Mott measurements reported in this paper, the absolute thicknesses provide information that is useful for comparisons with Mott work carried out by other researchers. The interferometric calibration method resulted in a conversion factor of 17.7 $\left(\mu \mathrm{g} / \mathrm{cm}^{2}\right) / \mathrm{kHz}$ with an uncertainty of $\pm 20 \%$. Thus the absolute thickness of the foils used are characterized by uncertainties of $\pm 21 \%$.

As illustrated in Fig. 1, the entire Mott-scattering chamber was isolated at high voltage. Therefore the detector outputs were converted to analog optical signals, coupled to ground potential through Lucite light pipes, and converted back to electrical pulses by photomultiplier tubes (PMT's). The PMT signals were routed to amplifier discriminators and subsequently counted by $10-\mathrm{MHz}$ scalers. Periodically, a 512-channel pulse-height analyzer (PHA) was used to monitor the PMT outputs. Based upon the electron-energy-loss spectrum displayed on the PHA, discriminator levels were chosen that optimally rejected inelastic events and yet still preserved the bulk of the elastic ones. The PHA was also used as a monitor of the electron beam energy through reference to the 62.5 and $84.5-\mathrm{keV}$ internal conversion peaks of the ${ }^{109} \mathrm{Cd}$ source.

The Mott polarimeter and its beam line were pumped by a liquid-nitrogen-trapped 6-in. oil diffusion pump located directly beneath the spin-rotator section. The vacuum chambers as well as the beam pipe were fabricated from aluminum, while internal components were constructed of materials that included brass, copper, stainless steel, Lucite, Teflon, and aluminum. In order to enhance the distinction between background electrons scattered from various surfaces and those scattered from the gold foils, all metallic components in the scattering chamber proper were fabricated from aluminum and where possible were coated with graphite. This exclusive use of low- $Z$ materials in the scattering chamber maximized the probability of energy loss in the spectrum of background electrons, thereby allowing the discriminators to be used effectively in the rejection of background events. The polarimeter pressure, as read by an ionization gauge at the spin rotator, was nominally $10^{-6}$ Torr.

Before the start of a polarization measurement, a beam of unpolarized electrons, produced by the removal of the linear polarizer from the Fano-source optical train, ${ }^{22,34}$ was guided into the Mott-scattering chamber. Phosphorescent $\mathrm{ZnS}$ viewing screens that could be moved in and out of the beam at the entrance and exit of the Wienfilter chamber were used to make preliminary adjustments of the beam alignment. With both screens removed, the beam was further tuned to maximize the counting rates in the surface barrier detectors and simultaneously minimize

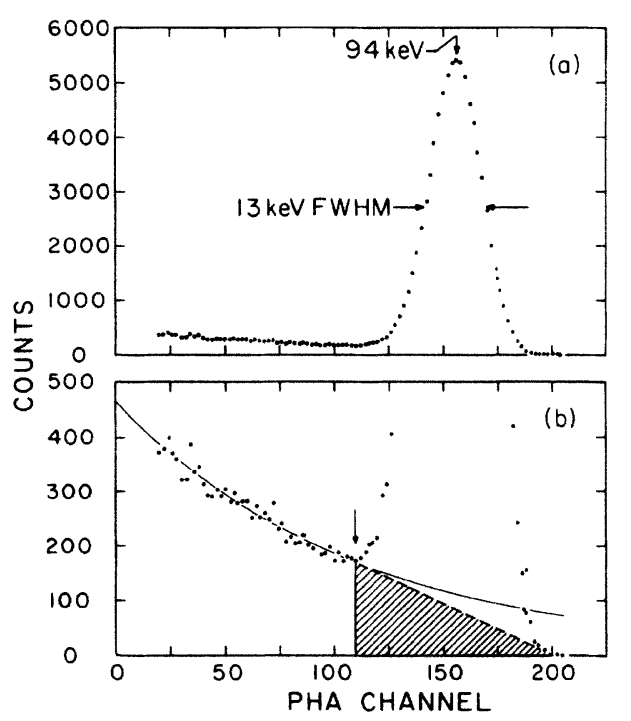

FIG. 5. Mott-scattering pulse-height-analyzer (PHA) spectrum. The vertical scale is a factor of 10 lower in (b) than in (a). The shaded area represents the "inelastic background" subtraction and the arrow indicates the discriminator threshold. The solid line gives the exponential fit to the inelastic scattering below threshold. Not shown at twice the elastic energy (188 $\mathrm{keV}$ ) is the pile-up spectrum that contains less than $0.2 \%$ of the counts above threshold.

the difference in the counting rates of the two detectors. Additional fine tuning was carried out with the polarized beam to make instrumental asymmetries resulting from beam misalignment virtually negligible. Typical pulseheight spectra for a gold foil and a bare Formvar foil are shown in Figs. 5 and 6, respectively. The 13-keV energy width of the elastic peak is attributable to the intrinsic resolution of the surface barrier detectors, a contention borne out by the presence of the seme width for the spectra of internal conversion electrons from the ${ }^{109} \mathrm{Cd}$ source. For each detector, a discriminator was set at the local minimum of the Mott spectrum with pulses above the discriminator threshold recorded in the $10-\mathrm{MHz}$ scalers, as described previously.

A complete Mott polarization measurement was carried out in the following manner. Counts from the two silicon surface barrier detectors were accumulated for both electron helicities, with typical counting times of $15 \mathrm{~s}$ for each

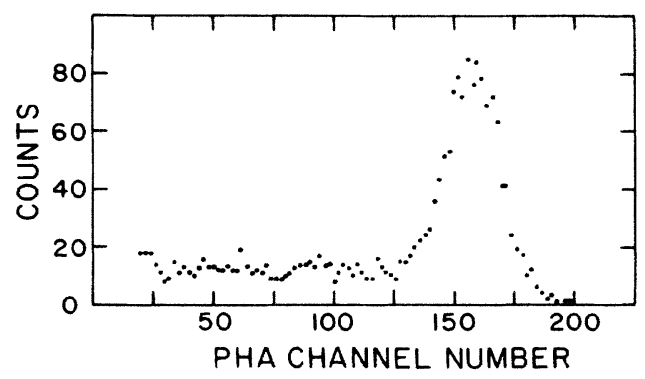

FIG. 6. Pulse-height spectrum for scattering from bare Formvar target. 
helicity. In order to avoid nonlinearities in the response of the photomultiplier tubes, counting rates were always kept below $2 \mathrm{kHz}$ through the insertion of a perforated screen in the uv light beam of the Fano source. After the electron polarization had been reversed three times, resulting in a total of approximately 160000 counts for the sum of the two detectors, a gold target of different thickness was moved into the beam position and the accumulation sequence was repeated. Once data for all four foils were obtained, the entire process was carried out three more times. A complete measurement including all experimental procedures and resulting in approximately $2.6 \times 10^{6}$ counts required no more than $30 \mathrm{~min}$.

\section{DATA ANALYSIS AND RESULTS}

The starting point for the extraction of the electron polarization $P$ from any real experimental measurement is the relation

$$
P=\Delta_{M} /\langle S\rangle \text {, }
$$

where $\langle S\rangle$ is the Sherman function, averaged over the solid-angle acceptance of the detectors, and $\Delta_{M}$ is the measured Mott asymmetry, defined by

$$
\Delta_{M}=\frac{N_{2}-N_{1}}{N_{2}+N_{1}}
$$

with $N_{1}$ and $N_{2}$ the accumulated counts from detectors 1 and 2 , respectively, resulting from single elastic scattering events from the gold target. The validity of Eq. (7) relies on the complete reflection symmetry of the Mott apparatus through the plane that contains the electron beam and that is perpendicular to the nominal scattering plane. It should be noted that complete reflection symmetry encompasses electronic as well as geometric properties of the polarimeter. Electronic symmetry, for example, requires that the efficiencies of the detectors be equal, that their energy resolutions be the same, and that their associated signal-processing hardware behave equivalently. The balance of this section of the paper is devoted to an examination of the effects that result in a departure from the ideal situation represented by Eq. (7), the corrections that must be made to account for these departures, and the implications that these departures have on the accuracy of a Mott measurement.

Throughout the subsequent discussion we will use the terms "inelastic" and "elastic" events. By the former we mean those events in the pulse-height spectrum that can be described by a function that decreases approximately exponentially with increasing energy, as shown in Fig. 5. By the latter we mean all remaining events that appear in the pulse-height spectrum at energies above the discriminator threshold. These definitions are purely operational and somewhat arbitrary, since (as we shall see) multipleand plural-scattering processes, inherently involving some degree of inelasticity, contribute measurably to our defined elastic spectrum. Conversely, incomplete energy deposition in the surface barrier detector by true elastically scattered electrons contribute to our "inelastic" background. Nonetheless, given the $13-\mathrm{keV}$ resolution of the detectors, there is probably no simple, unambiguous way of labeling the principal sections of the pulse-height spectrum.

Having explained our use of terminology, we now list the dominant effects that potentially can contribute to departures from the ideal case of Eq. (7).

(1) Detector and electronic noise contributions to $N_{1}$ and $N_{2}$.

(2) Inelastic contributions to $N_{1}$ and $N_{2}$, resulting from the finite detector resolution and attributable to (a) single, multiple, and plural scattering ${ }^{16,55-60}$ from both gold and the Formvar, (b) target scattering combined with wall reflections, ${ }^{6,61}$ and (c) incomplete energy deposition in the detector due to outscattering from the detector. ${ }^{62}$

(3) Elastic contributions to $N_{1}$ and $N_{2}$ that are degraded in asymmetry, resulting from (a) plural and multiple scattering, (b) target scattering combined with wall reflections, and (c) Formvar scattering.

(4) Instrumental asymmetries, including unequal detector efficiencies.

We illustrate schematically in Fig. 7 the influence of several of these effects on the pulse-height spectrum.

As can be seen from the figure, the use of discriminators virtually eliminates the effect of noise on our counting rates. Quantitatively, we found that above the discriminator threshold, the "dark-counting" rate that remained when the electron beam was blocked was always less than $0.1 \%$ of the Mott counting rate produced by the incident polarized electron beam. Therefore, in our analysis we neglected all noise corrections to $N_{1}$ and $N_{2}$.

Unfortunately, the processes included in the second and third categories on our list were more significant. For several of them we were able to make corrections relatively easily; in other cases the task was quite difficult, if not

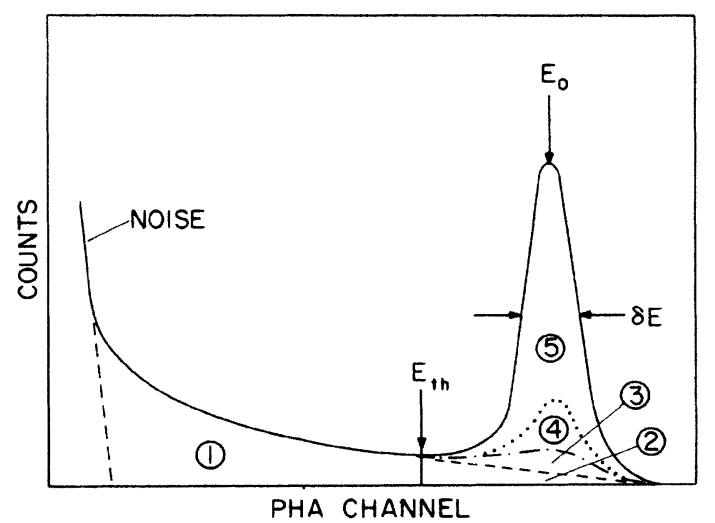

FIG. 7. Schematic representation of contributions to a typical Mott pulse-height spectrum, showing incident electron energy $E_{0}$, detector energy resolution (full-width-half-maximum) $\delta E$, and discriminator threshold $E_{\mathrm{th}}$. Events in regions (1) and (2) comprise the "inelastic background"; events in region (3) are due to elastic scattering from Formvar; events in region (4) are due to "inelastic" multiple and plural scattering; events in region (5) are due to elastic single scattering. Events in region (1) are eliminated by the discriminator, while those in regions (2) and (3) are removed in the background subtraction process. 
impossible without the introduction of significant uncertainties. As an example of a relatively simple, straightforward correction, we cite the influence of both elastic and inelastic scattering from Formvar, which we were able to handle by subtraction of the rate produced by scattering from the bare Formvar target foil, a procedure that we will discuss in detail shortly. A considerably more complicated correction is that required for the effects of elastic multiple and plural scattering, which we treated by extrapolating the Mott asymmetry to zero foil thickness by a procedure that we will also discuss in detail.

Before proceeding to these discussions, however, we first consider the effects of instrumental asymmetries, the last category on our list. In principle these effects can be eliminated by reversing the polarization vector of the incident electron beam, provided the reversal itself does not introduce an asymmetry. Modifying Eq. (8) to account for the effective efficiency of the detectors (including both geometric and electronic factors) we can write under these circumstances

$$
\Delta_{M}= \pm \frac{\alpha_{2} N_{2}^{ \pm}-\alpha_{1} N_{1}^{ \pm}}{\alpha_{2} N_{2}^{ \pm}+\alpha_{1} N_{1}^{ \pm}},
$$

where the superscript \pm refers to the two directions of the polarization vector, and $\alpha_{i}$ is the effective efficiency of detector $i$. With $\beta=\alpha_{1} / \alpha_{2}$ and $R^{ \pm}=N_{1}^{ \pm} / N_{2}^{ \pm}$, we can rewrite Eq. (9) as

$$
\Delta M= \pm \frac{1-\beta R^{ \pm}}{1+\beta R^{ \pm}},
$$

or, solving for $\beta R^{ \pm}$, we have

$$
\beta R^{ \pm}=\frac{1 \mp \Delta_{M}}{1 \pm \Delta_{M}} .
$$

By constructing the ratio $\beta R^{+} / \beta R^{-}$from measurements using opposite directions of polarization, we may solve for $\Delta_{M}$, obtaining

$$
\Delta_{M}=\frac{1-\xi}{1+\xi},
$$

where

$$
\xi=\left(\frac{N_{1}^{+} N_{2}^{-}}{N_{2}^{+} N_{1}^{-}}\right)^{1 / 2} .
$$

Since $\xi$ is independent of $\beta, \Delta_{M}$ obtained from Eq. (12) is independent of instrumental asymmetries. In carrying out our measurements, we utilized this fact and thus effectively eliminated the influence of instrumental asymmetries. Since the reversal of the polarization vector relied on an optical reversal of the uv circular polarization in the Fano source, and since applications of the source to electronhydrogen scattering ${ }^{34}$ revealed an absence of systematic asymmetries related to reversal at a level of less than $10^{-3}$, we are confident that the reversal process did not introduce any systematic errors at the level of sensitivity of our Mott measurements.

We now proceed to the more difficult corrections. In order to eliminate the effects of inelastic background events, we adopted a two-step procedure. First we set the discriminator levels on each detector channel to prevent counting of pulses with amplitudes below a value corresponding to the "knee" of the spectrum shown in Fig. 5. For the counted pulses, we made a further background correction by determining the fraction of those counts that are typically contained in a triangular region extending from the knee of the spectrum to the high-energy tail of the elastic peak as shown in Fig. 5. We then reduced the accumulated scaler counts by this fraction. We adopted this relatively unsophisticated triangular subtraction procedure because we believe that more elaborate techniques are not justified, given our poor knowledge of the true energy distribution of the inelastic events. ${ }^{63-72}$ Moreover we found that for a given combination of detector and direction of polarization, the fraction of counts in the triangular region remained constant from run to run, within counting statistics. Thus, denoting the total number of counts above threshold by $\left(N_{i}^{ \pm}\right)_{d}$ and the background fraction by $b_{i}^{ \pm}$we can express the corrected elastic count number $\left(N_{i}^{ \pm}\right)_{e}$ as

$$
\left(N_{i}^{ \pm}\right)_{e}=\left(N_{i}^{ \pm}\right)_{d}\left(1-b_{i}^{ \pm}\right)=\left(N_{i}^{ \pm}\right)_{d}-B_{i}^{ \pm},
$$

where the subscript $i=1,2$ specifies the detector and $B_{i}^{ \pm}=\left(b_{i}^{ \pm}\right)\left(N_{i}^{ \pm}\right)_{d}$. The four inelastic background fractions, $b_{1}^{ \pm}$and $b \frac{ \pm}{2}$, are given for each of the four gold-foil thicknesses in Table I along with the fraction of events, $b_{F}$, attributed to scattering from Formvar which we will discuss shortly. As can be seen, each of the inelastic background fractions is less than or equal to $6 \%$ and, within counting statistics, is independent of foil thickness.

In an effort to understand in more detail the contribution of various processes to the triangular tail of the in-

TABLE I. Background fractions $b_{i}^{ \pm}$, due to the inelastic scattering ("triangle" subtraction), and $b_{F}$, due to elastic Formvar scattering, for various thicknesses of gold foils.

\begin{tabular}{cccccc}
\hline $\begin{array}{c}\text { Target } \\
\text { thickness } \\
\left(\mu \mathrm{g} / \mathrm{cm}^{2}\right)\end{array}$ & $\begin{array}{c}b_{1}^{+} \\
(\%)\end{array}$ & $\begin{array}{c}b_{1}^{-} \\
(\%)\end{array}$ & $\begin{array}{c}b_{2}^{+} \\
(\%)\end{array}$ & $\begin{array}{c}b_{2}^{-} \\
(\%)\end{array}$ & $\begin{array}{c}b_{F} \\
(\%)\end{array}$ \\
\hline 62 & $6.0(5)$ & $4.8(4)$ & $4.2(4)$ & $4.9(5)$ & $1.4(1)$ \\
53 & $5.2(7)$ & $5.0(6)$ & $4.0(5)$ & $4.7(7)$ & $1.9(1)$ \\
44 & $5.6(8)$ & $4.0(5)$ & $3.9(5)$ & $4.7(7)$ & $2.4(1)$ \\
27 & $5.5(10)$ & $3.4(6)$ & $4.2(7)$ & $3.7(7)$ & $4.6(2)$ \\
All & $5.7(4)$ & $4.4(3)$ & $4.1(2)$ & $4.6(3)$ & \\
Targets & & & & & \\
\hline \hline
\end{tabular}


TABLE II. Possible contributions to the inelastic background fractions $b_{i}^{ \pm}$and their expected dependence on $\Delta_{M}$ and gold target thickness. The dependence given in the fourth column is obtained using the assumptions in the third column together with Eq. (15).

\begin{tabular}{|c|c|c|c|}
\hline Contribution & $\begin{array}{l}\text { Dependence for } \\
\text { increasing } \\
\text { gold thickness }\end{array}$ & $\begin{array}{l}\text { Assumed } \\
\text { dependence } \\
\text { of } \Delta_{M}^{B}\end{array}$ & $\begin{array}{c}\text { Dependence of } \\
\left(b_{2}^{+} b_{1}^{-} / b_{1}^{+} b_{2}^{-}\right)^{1 / 2} \\
\text { on } \Delta_{M}\end{array}$ \\
\hline Noise & Decreasing & 0 & $\sim \frac{1-\Delta_{M}}{1+\Delta_{M}}$ \\
\hline $\begin{array}{c}\text { Detector } \\
\text { outscattering }\end{array}$ & Constant & Equal to $\Delta_{M}$ & Independent of $\Delta_{M}$ \\
\hline $\begin{array}{l}\text { Inelastic multiple } \\
\text { and plural } \\
\text { scattering }\end{array}$ & Increasing & 0 & $\sim \frac{1-\Delta_{M}}{1+\Delta_{M}}$ \\
\hline $\begin{array}{l}\text { Target scattering } \\
\text { plus wall } \\
\text { reflection }\end{array}$ & Constant & 0 & $\sim \frac{1-\Delta_{M}}{1+\Delta_{M}}$ \\
\hline
\end{tabular}

elastic spectrum, we considered their expected dependence on foil thickness and asymmetry. Table II lists these contributions and their expected dependences, the latter based upon the assumptions that $\left(N_{i}^{ \pm}\right)_{d}$ increases with thickness and that

$$
\begin{aligned}
\left(\frac{b_{2}^{+}}{b_{1}^{+}} \frac{b_{1}^{-}}{b_{2}^{-}}\right)^{1 / 2} & =\left[\frac{\left(N_{1}^{+}\right)_{d} /\left(N_{2}^{+}\right)_{d}}{B_{1}^{+} / B_{2}^{+}} \frac{\left(N_{2}^{-}\right)_{d} /\left(N_{1}^{-}\right)_{d}}{B_{2}^{-} / B_{1}^{-}}\right)^{1 / 2} \\
& \approx \frac{\left(1-\Delta_{M}\right) /\left(1+\Delta_{M}\right)}{\left(1-\Delta_{M}^{B}\right) /\left(1+\Delta_{M}^{B}\right)}
\end{aligned}
$$

where $\Delta_{M}^{B}$ is the Mott asymmetry associated with the triangular background events. The second, approximate equality of Eq. (15) holds because of the approximate relation

$$
\frac{\left(N_{1}^{+}\right)_{d}\left(N_{2}^{-}\right)_{d}}{\left(N_{2}^{+}\right)_{d}\left(N_{1}^{-}\right)_{d}} \approx \frac{\left(N_{1}^{+}\right)_{e}\left(N_{2}^{-}\right)_{e}}{\left(N_{2}^{+}\right)_{e}\left(N_{1}^{-}\right)_{e}} .
$$

From the observation in each case that $b_{i}^{ \pm}$is independent of target thickness we conclude that only detector outscattering and target scattering plus wall reflections actually contributed to the triangular background. Furthermore, from the expected dependence of $\left(b_{2}^{+} b_{1}^{-} / b_{1}^{+} b_{2}^{-}\right)^{1 / 2}$ on $\Delta_{M}$ we can calculate the relative magnitude of these two contributions. If we set up the relation

$$
\left(\frac{b_{2}^{+} b_{1}^{-}}{b_{2}^{-} b_{1}^{+}}\right)^{1 / 2}=C+(1-C)\left(\frac{1-\Delta_{M}}{1+\Delta_{M}}\right)
$$

with $C$ an unknown parameter, we find using the values of $b_{i}^{ \pm}$from Table II together with a typical value of 0.24 for $\Delta_{M}$ that $C \approx 0.5$. Thus we conclude that detector outscattering and target scattering plus wall reflections contribute almost equally.

While contributions to the inelastic tail from detector outscattering are due, in fact, to "good elastic events," the contribution of such events to the individual background fractions, $b_{i}^{ \pm}$, is difficult to assess with any accuracy.
Therefore we have chosen to classify all of these events as background. Such a classification, in principle, does not affect the value of the Mott asymmetry.

In order to determine the contribution of elastic scattering arising from the Formvar backing of the gold targets, we compared the counting rate for scattering of polarized electrons from a bare Formvar target with the rates from each of the gold targets for the same incident electron current. As expected for a low- $Z$ material, we found that the analyzing power of the Formvar is essentially zero. Consequently, for each of the gold foils we determined the fraction $\left(b_{F}\right)_{i}^{\dagger}$ of electrons elastically scattered from the Formvar backing from the relation

$$
\left(b_{F}\right)_{i}^{ \pm}=\frac{2 N_{F}}{\left(N_{1}^{ \pm}\right)_{e}+\left(N_{2}^{ \pm}\right)_{e}},
$$

where $N_{F}$ is the number of elastic events from Formvar registered at either detector. The freedom to choose either detector resulted from our observation that with a properly tuned beam, $N_{F}$ was independent of the choice of detector. Since Formvar has zero analyzing power, we also found that $N_{F}$ was independent of polarization direction. We further found that the sum $\left(N_{1}^{ \pm}\right)_{e}+\left(N_{2}^{ \pm}\right)_{e}$ was independent of polarization direction under proper tuning conditions. Thus in Table I we present $\left(b_{F}\right)_{i}^{ \pm}$as a function of foil thickness only and omit the $i$ subscript and \pm superscript. The fraction $b_{F}$ has a maximum value of 4.6\% for the thinnest gold foil, and hence the elastic event rate due to scattering from Formvar is equivalent to that for a gold foil $1.2 \mu \mathrm{g} / \mathrm{cm}^{2}$ thick.

The corrected Mott event rate, $\left(N_{i}^{ \pm}\right)_{c}$, with background subtractions made for both inelastic and elastic Formvar scattering, is given by

$$
\left(N_{i}^{ \pm}\right)_{c}=\left(N_{i}^{ \pm}\right)_{d}-B_{i}^{ \pm}-N_{F},
$$

or alternatively by

$$
\left(N_{i}^{ \pm}\right)_{c}=\left(N_{i}^{ \pm}\right)_{d}\left(1-b_{i}^{ \pm}\right)\left(1-f_{i}^{ \pm}\right),
$$

where 


$$
f_{i}^{ \pm}=\frac{N_{F}}{\left(N_{i}^{ \pm}\right)_{e}}=\frac{b_{F}\left[\left(N_{1}^{ \pm}\right)_{e}+\left(N_{2}^{ \pm}\right)_{e}\right]}{2\left(N_{i}^{ \pm}\right)_{e}} .
$$

We can combine these event rates to form a quantity $\xi_{c}$ analogous to $\xi$ of Eq. (13) with the qualification that $\xi_{c}$ now depends on foil thickness. We thus have the corrected Mott asymmetry for foil thickness $t$,

$$
\Delta_{M}(t)=\frac{1-\xi_{c}(t)}{1+\xi_{c}(t)}
$$

with $\xi_{c}(t)$ given by

$$
\begin{aligned}
\xi_{c}(t)= & {\left[\frac{\left(N_{1}^{+}\right)_{c}\left(N_{2}^{-}\right)_{c}}{\left(N_{1}^{-}\right)_{c}\left(N_{2}^{+}\right)_{c}}\right]^{1 / 2} } \\
= & {\left[\frac{\left(N_{1}^{+}\right)_{d}\left(N_{2}^{-}\right)_{d}}{\left(N_{1}^{-}\right)_{d}\left(N_{2}^{+}\right)_{d}}\right]^{1 / 2}\left[\frac{\left(1-b_{1}^{+}\right)}{\left(1-b_{1}^{-}\right)} \frac{\left(1-b_{2}^{-}\right)}{\left(1-b_{2}^{+}\right)}\right]^{1 / 2} } \\
& \times\left[\frac{\left(1-f_{1}^{+}\right)}{\left(1-f_{1}^{-}\right)} \frac{\left(1-f_{2}^{-}\right)}{\left(1-f_{2}^{+}\right)}\right]^{1 / 2} .
\end{aligned}
$$

From the measurements of the inelastic background fractions $b_{i}^{ \pm}$, we determined the second bracketed term of the lower equality of Eq. (23) to be $0.991(3)$. We found that the third term varied from $0.974(5)$ for the $27 \mu \mathrm{g} / \mathrm{cm}^{2}$ target to $0.993(1)$ for the $62 \mu \mathrm{g} / \mathrm{cm}^{2}$ target.

The foregoing analysis implies that multiple and plural scattering contribute negligibly to the inelastic background fractions $b_{i}^{ \pm}$, determined from our triangular subtraction method. We might therefore be tempted to conclude that multiple and plural scattering have only a small effect on our measurements, were it not for the common knowledge $^{16-25,32,33}$ that they are the primary cause of the decrease in the Mott asymmetry (or effective Mott analyzing power) as the foil thickness increases, the incident electron energy decreases, or the scattering angle $\theta$ increases. We must thus examine the elastic portion of the spectrum in a search for the presence of multiple- and plural-scattering events. Consistent with our prior notation, we refer to these as "elastic multiple- and pluralscattering" contributions, despite the fact that they are almost certainly associated with some energy loss, a subject we will consider in the last section of this paper.

In order to evaluate the possible influence of elastic multiple and plural scattering on our measurements, we carry out an extrapolation of our data to zero foil thickness, where by definition only single scattering may occur. While foil thickness extrapolation is a general procedure that is widely used, an examination of Mott literature reveals that the specifics of the extrapolation vary considerably. We therefore carry out the extrapolation by a number of different techniques, all of which we believe are equally justifiable based upon present theoretical knowledge of multiple and plural scattering.

Although considerable theoretical attention has been devoted to the theory of multiple and plural scattering, ${ }^{16,55-60}$ our starting point is the expansion of $N(\theta, \phi)$, the scattered intensity, in powers of $t$, the target thickness. For the thin targets employed in our studies, truncation of the expansion at second order in $t$ should suffice. In the case of electrons with a transverse polarization $P$, it has been shown ${ }^{16}$ that $N(\theta, \pm \pi / 2)$ can be expressed as

$N(\theta, \pm \pi / 2) \approx \rho t I(\theta)\{(1+\beta t)[1 \pm P S(\theta)] \mp \alpha t P S(\theta)\}$,

where $\rho$ is the target density, $I(\theta)$ is the spin-independent differential cross section, and $\alpha$ and $\beta$ are parameters that contain logarithmic dependences on $t$. Generally, the dependence of $\alpha$ and $\beta$ on $t$ is ignored and both are treated as unknown coefficients, to be determined from fitting procedures applied to the Mott data. With the use of Eqs. (5) and (24) we find for the thickness-dependent ideal Mott asymmetry $\delta_{M}(t)$ the expression

$$
\delta_{M}(t) \approx \frac{\delta_{M}(0)[1+t(\beta-\alpha)]}{1+\beta t},
$$

where $\delta_{M}(0)=\delta_{M}(t=0)=P S(\theta)$. Using Eq. (25) as the basic relation for the thickness dependence of the Mott asymmetry, we can develop several simplified operational approaches to the extrapolation to zero foil thickness. Expanding the inverse of $\delta_{M}(t)$ in powers of $t$ and keeping only terms to second order consistent with Eq. (23), for example, yields

$$
\frac{1}{\delta_{M}(t)} \approx \frac{1}{\delta_{M}(0)}(1+\alpha t),
$$

provided the quantity $(\beta-\alpha) t$ is small compared to unity. The approach suggested by Eq. (26) has been used in a number of previous studies. $6,16,21,22,50-53$ It should be observed, however, that in this approach only the coefficient $\alpha$ survives, which for $\theta>90^{\circ}$ contains depolarizing effects dominated by large-angle double scattering rather than small-angle multiple scattering.

In order to gain a measure of the relative strength of double scattering to that of single scattering, we must look at the coefficient $\beta$, which we can obtain by plotting $N_{\text {tot }} / t$ as a function of $t$, where $N_{\text {tot }}$ is given by

$$
N_{\text {tot }}=N_{2}(\theta)+N_{1}(\theta)=2 \rho I(\theta) t(1+\beta t) .
$$

Using our corrected event rates $\left(N_{1}^{+}\right)_{c}$ and $\left(N_{2}^{+}\right)_{c}$ [or equivalently $\left(N_{1}^{-}\right)_{c}$ and $\left.\left(N_{2}^{-}\right)_{c}\right]$ for $N_{1}(\theta)$ and $N_{2}(\theta)$, respectively, we show in Fig. 8(a) an example of such a plot, from which we find $2 \rho I=0.035(10) \mathrm{cm}^{2} / \mu \mathrm{g}$ and $\beta=0.0156(35) \mathrm{cm}^{2} / \mu \mathrm{g}$. Thus, our elastic spectra contain significant contributions from double scattering for foils as thin as $44 \mu \mathrm{g} / \mathrm{cm}^{2}$.

Returning to consideration of extrapolation methods for $\delta_{M}(t)$ we observe that to first order in $t$, the form

$$
\delta_{M}(t)=\delta_{M}(0) e^{-\alpha t}
$$

is the same as that given by Eq. (26). For foils that are sufficiently thin, Eq. (28) may be a sufficiently good representation to use for thickness extrapolation. It has, in fact, been successfully employed in many Mott measurements, either as stated ${ }^{24}$ or with $e^{-\alpha t}$ taken as $(1-\alpha t) .17,18,20,21,27,30,33$

The forms given by Eqs. (26) and (28), by virtue of the approximations used, may not be the best representations for $\delta_{M}(t)$, an assertion borne out by at least some of our 
data. In an effort to develop alternate if not more accurate extrapolation methods, we are thus led to consider relations that retain the information contained in the coefficient $\beta$. By combining Eqs. (25) and (27) and retaining terms to first order in $N_{\text {tot }}(t)$, we find, for example, the relations

$$
\delta_{M}(t)=\delta_{M}(0)\left[1-\frac{\alpha}{2 \rho I(\theta)} N_{\text {tot }}(t)\right)
$$
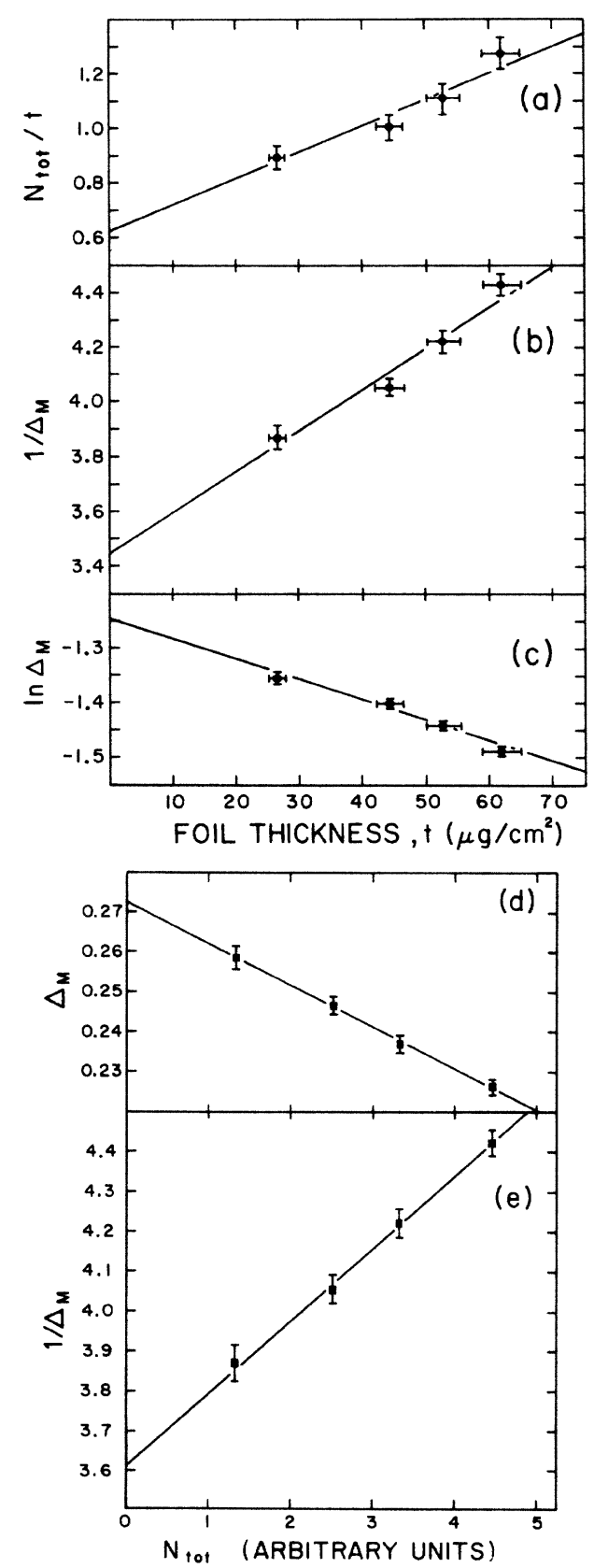

FIG. 8. (a) Plot of $N_{\text {tot }} / t$ as a function of $t$ illustrating the quadratic dependence of $N_{\text {tot }}$ on $t$. (b) Extrapolation of $1 / \Delta_{M}$ to zero foil thickness, in accordance with Eq. (26). (c) Extrapolation of $\ln \left(\Delta_{M}\right)$ to zero foil thickness, in accordance with Eq. (28). (d) Extrapolation of $\Delta_{M}$ to zero $N_{\text {tot }}$, in accordance with Eq. (29). (e) Extrapolation of $1 / \Delta_{M}$ to zero $N_{\text {tot }}$, in accordance with Eq. (30). and

$$
\frac{1}{\delta_{M}(t)}=\frac{1}{\delta_{M}(0)}\left(1+\frac{\alpha}{2 \rho I(\theta)} N_{\mathrm{tot}}(t)\right) .
$$

While other usable expressions can probably be developed, it is instructive at this point to consider the relative merits of the four forms contained in Eqs. (26) and (28)-(30), and to see whether, in the absence of additional theoretical guidance, any one of them is substantially better, based upon its ability to fit to the data.

Before applying any of the four forms to the data, we first note that with the substitution of the corrected Mott event rates $\left(N_{i}^{ \pm}\right)_{c}$ for the scattered intensities $N_{i}(\theta)$ as previously described, we may replace $\delta_{M}(t)$ by the actual Mott asymmetry, $\Delta_{M}(t)$, given by Eq. (22). Proceeding in this manner, we can generate the four extrapolations shown graphically in Figs. 8(b)-8(d), corresponding respectively to Eqs. (26) and (28)-(30). These graphs, together with their least-squares fits for $\Delta_{M}(0)$ and $\alpha$, summarized in Table III, represent results for one of our two data sets. (Since the other data set produces similar results, we have not displayed them.) It should be noted that while the values of $\alpha$ associated with the $t$ extrapolations are obtained from those extrapolations directly, the values of $\alpha$ associated with the $N_{\text {tot }}$ extrapolations rely additionally on the magnitude of $2 \rho I$ obtained from the plot of $N_{\text {tot }} / t$ versus $t$ shown in Fig. 8(a).

Several observations are now in order. (1) From the summary of Table III, there is an apparent discrepancy between the values of $\alpha$ obtained from the $N_{\text {tot }}$ extrapolations on one hand and those obtained from the $t$ extrapolations on the other. This discrepancy is a natural consequence of the retention of a $t^{2}$ term in $N_{\text {tot }}$, which is absent by definition in the $t$ extrapolations. (2) Based upon the $t$ extrapolations carried out for our 94-keV Mott measurements, including the two corresponding to the data set not displayed, we obtain an average value of $0.0034(7)$ $\mathrm{cm}^{2} / \mu \mathrm{g}$ for the coefficient $\alpha$, the $\pm 21 \%$ uncertainty dominated by the $\pm 20 \%$ uncertainty in the interferometric foil-thickness calibration. From earlier measurements carried out at a scattering energy of $100 \mathrm{keV}$ we obtain (after recent revisions) a value of $0.0028(10) \mathrm{cm}^{2} / \mu \mathrm{g}$ for $\alpha$, based upon the $t$ extrapolation method given by Eq. (26). We observe that both of these $\alpha$ values are in good agreement with those measured by other researchers. ${ }^{18,21,61,73}$ (3) Based upon the uncertainties in $\Delta_{M}(0)$ given in Table III for each of the four extrapolation methods, it would appear that the $N_{\text {to }}$ extrapolations provide a somewhat better approach to the problem than the $t$ extrapolations. Nonetheless, since the $t$ extrapolations still provide reasonable $\chi^{2}$ values, and since there is no strong physics justification for using the $N_{\text {tot }}$ method, save its inclusion of some (but clearly not all) of the inherent $t^{2}$ dependence, we believe that it is improper to reject the $t$ extrapolation results summarily.

In light of the preceding observations we have concluded that a reasonable way to calculate a final value of $\Delta_{M}(0)$ is to use a weighted average of the four extrapolations. In this way we lend more credence to the $N_{\text {tot }}$ extrapolations without rejecting the $t$ extrapolations com- 
TABLE III. Results of the Mott asymmetry extrapolations to zero foil thickness shown in Figs. $8(\mathrm{~b})-8(\mathrm{e})$ for one polarization measurement at $94 \pm 2 \mathrm{keV}$.

\begin{tabular}{lccccc}
\hline \hline $\begin{array}{c}\text { Method of } \\
\text { extrapolation }\end{array}$ & Eq. & $\Delta_{M}(0)$ & $\alpha\left(\mathrm{cm}^{2} / \mu \mathrm{g}\right)$ & $\chi^{2}$ per deg. freedom & $\begin{array}{c}\chi^{2} \text { confidence } \\
\text { level }\end{array}$ \\
\hline $1 / \Delta_{M}$ vs $t$ & $(26)$ & $0.2910(83)$ & $0.0044(11)$ & 1.06 & $35 \%$ \\
$\ln \Delta_{M}$ vs $t$ & $(28)$ & $0.2868(69)$ & $0.0037(9)$ & 0.94 & $39 \%$ \\
$\Delta_{M}$ vs $N_{\text {tot }}$ & $(29)$ & $0.2723(36)$ & $0.0013(4)$ & 0.09 & $92 \%$ \\
$1 / \Delta_{M}$ vs $N_{\text {tot }}$ & $(30)$ & $0.2767(45)$ & $0.0018(5)$ & 0.13 & $88 \%$ \\
Weighted average & & $0.2772(69)$ & & & \\
\hline \hline
\end{tabular}

The uncertainty has been increased to include within $2 \sigma$ the four values of $\Delta_{M}(0)$ determined from the separate extrapolations.

pletely. Since all the extrapolation procedures are fraught with some degree of theoretical uncertainty, however, we believe that conservatism dictates an assignment of a onestandard-deviation $(\sigma)$ uncertainty to the weighted average $\Delta_{M}(0)$ such that all four individual extrapolation results fall within $\pm 2 \sigma$ of $\Delta_{M}(0)$. For the data set summarized in Table III, such an assignment results in a onestandard-deviation fractional uncertainty of $\pm 2.5 \%$. On the basis of our other measurements, however, we believe that a value of $\pm 3 \%$ might have more general applicability.

In order to complete the Mott analysis, we use Eq. (7) with $\Delta_{M}(0)$ substituted for $\Delta_{M}$. For $\langle S\rangle$ we use the value of $-0.387(8)$ which we obtain from interpolated theoretical calculations of other researchers. (We rely on theoretical values of $\langle S\rangle$ rather than experimental ones, since the latter were obtained from difficult double-scattering experiments that are susceptible to a number of systematic errors.) The $\pm 2 \%$ fractional error in $\langle S\rangle$ results from uncertainties in screening corrections and the absence of precise double-scattering experiments that might provide an empirical value for $\langle S\rangle$. Since the uncertainty in $\langle S\rangle$ is essentially a systematic one, we combine the fractional uncertainties in $\Delta_{M}(0)$ and $\langle S\rangle$ linearly, in which case we obtain a one-standard-deviation precision of $\pm 5 \%$ for $P$. While not central to the main purpose of this paper, we point out for completeness that with this analysis we obtain a value of $P=0.716(32)$ for the polarization of the beam used in the Mott measurements summarized in Table III.

\section{CONCLUSIONS}

The analysis and results described in the preceding section lead us to three significant conclusions. First, above the discriminator threshold, which was set at a value corresponding to a $24 \mathrm{keV}$ energy loss for the scattered electrons, we find that plural- and/or multiple-scattering effects are concentrated in the elastic peak rather than in the extrapolated inelastic triangular tail. Second, within the extrapolated inelastic tail, the events are equally divided between those resulting from the energy-loss processes associated with detector outscattering on one hand and with forward scattering from the target followed by large angle wall collisions on the other. Third, at the present time, as a consequence of the foil-thickness dependence of the Mott asymmetry and the theoretical uncertainties in the calculations of the Sherman function, ${ }^{6,46}$ conventional Mott polarization measurements appear to be limited to an absolute precision of $\pm 5 \%$.

We now examine the implications of these conclusions for the physics of multiple and plural scattering (insofar as it pertains to Mott scattering) as well as for the claimed precision of a number of published Mott studies. For the purpose of this discussion, we will limit ourselves to the case of double scattering, a restriction that is justified for thin targets, as evidenced by the quadratic dependence of $N_{\text {tot }}$ on $t$ illustrated in Fig. 8(a).

It is obvious that double-scattering processes can contribute substantially to the total event rate only when the cross sections associated with both scatterings are relatively high. Recently, we learned ${ }^{74}$ that much conventional thought holds that only inelastic events characterized by large energy losses $(\Delta E \sim 1 \mathrm{keV})$ meet this criterion. We speculate that such a body of opinion may have developed because the scale of the energy loss of a typical inelastic event is sometimes mistakenly associated with the value of the mean atomic-excitation energy, $I$, that appears in the Bethe-Block equation ${ }^{75}$ for the electron stopping power, $-d E / d x$. In the case of gold, an element with the relatively large atomic number of $Z=79, I$ indeed has a value of approximately $800 \mathrm{eV}$, as calculated from the semiempirical relation ${ }^{75}$

$$
I \sim 9.1 Z\left(1+1.9 Z^{-2 / 3}\right) \mathrm{eV},
$$

which is valid for $Z \geq 4$. The association of $I$ with the energy loss of a typical inelastic event, however, is improper, as we shall now demonstrate.

By definition, $I$ represents the average of all possible atomic-excitation energies weighted by the cross section associated with each corresponding energy loss. In order to arrive at a value for $I$ of $800 \mathrm{eV}$, given the much larger energies associated with inner-shell excitations (for example, $7.5 \times 10^{4} \mathrm{eV}$ for the $K$ shell), it is obvious that lowenergy outer-shell excitations must be heavily represented in the double-scattering process. Thus, we should expect double-scattering events to make their presence felt only in a rather narrow band of energies below the elastic value, a conclusion that is borne out by our first result, to wit we find no evidence of a foil thickness dependence of the inelastic triangle, a part of the exponential spectral section that is dominated by large energy losses. (A simi- 
lar inference may be drawn from the data shown in Fig. 3 of Ref. 25.)

In an effort to verify the conclusion of our naive analysis we searched the literature for reports of experimental measurements of energy-loss spectra for approximately $100-\mathrm{keV}$ electrons scattered into large angles by high- $Z$ atoms. Unfortunately, and surprisingly, the literature on the subject, both theoretical and experimental, is rather sparse. ${ }^{63-72}$ Nonetheless, our search revealed three particularly relevant experimental reports ${ }^{66,71,72}$ covering incident electron energies from $30 \mathrm{keV}$ to $3 \mathrm{MeV}$ and scattering angles from $20^{\circ}$ to $160^{\circ}$ for thin foil targets consisting of carbon, copper, and gold. For incident energies between 40 and $100 \mathrm{keV}$ and for scattering angles greater than $90^{\circ}$, where Møller scattering ${ }^{76}$ (scattering from free electrons) is kinematically forbidden, the energy-loss spectra for copper and gold targets are characterized by inelastic cross sections that fall by several orders of magnitude $^{71,72}$ (from the elastic value) as the energy loss, $\Delta E$, increases from 0 to $2 \mathrm{keV}$. The cross sections begin to rise $^{66,72}$ again only after $\Delta E$ reaches $20-40 \mathrm{keV}$. Although double scattering may have degraded the results somewhat, it appears that for $100-\mathrm{keV}$ electrons incident on copper, the inelastic cross section decreases by almost a factor of 100 by the time $\Delta E$ reaches $500 \mathrm{eV}$. In fact, Grachev et $a .^{72}$ report that "the intensity near the elastic peak is well approximated by a $\ldots(\Delta E)^{-2}$ law" for scattering angles of $30^{\circ}, 45^{\circ}, 60^{\circ}$, and $90^{\circ}$.

The body of inelastic data is thus completely consistent with our own observations and with the conclusions of our naive analysis. We note that the theoretical problem of large-angle scattering with small energy loss for approximately $100-\mathrm{keV}$ incident energies is a complex one and clearly requires additional work, since existing calculations fall far short of explaining the experimental results. Additionally experimental measurements, in which foil targets are replaced by atomic beams, would further elucidate the problem by removing ambiguities associated with plural scattering.

We now turn to the impact of our studies on other Mott measurements. Increasingly, papers have appeared in which Mott asymmetries and even absolute values of electron polarization are quoted with fractional uncertainties below $\pm 1 \%$. In some instances the claim is explicit, as in the case of Campbell et al. ${ }^{23}$ while in others it is implicit, as in the case of two very recent letters by Allenspach et al. ${ }^{36}$ and by Alvarado et al. ${ }^{77}$ (We wish it to be understood that we are not singling out these authors for criticism, since they are only part of a widespread trend.) We believe that such claims are misleading and totally unjustified, in light of both the double-scattering problem and the theoretical uncertainties in the calculation of the Sherman function itself. ${ }^{6,46}$ We urge all authors to clearly state that their small uncertainties apply to relative values of the polarization only.

We note further that some workers have tacitly assumed that the new compact Mott polarimeters ${ }^{25,32,33}$ are completely free from foil-thickness extrapolation problems by virtue of their far superior energy resolution. In view of the concentration of the inelastic cross section to a region of small $\Delta E$, such assumptions must be made with great care. For example, estimates indicate ${ }^{78}$ that the Rice cylindrical Mott polarimeter is probably characterized by a resolution of approximately $10 \mathrm{eV}$, and the spherical version (operating at the much lower incident energies of 20 to $40 \mathrm{keV}$ ) is probably characterized by a resolution of less than $50 \mathrm{eV}$. While double scattering, and hence foil-thickness extrapolations, may be less of a problem for these devices than for conventional polarimeters, we believe that the compact Mott devices still suffer from residual foil-thickness effects. In this context we point out that foil-thickness extrapolations must be carried out cautiously, with attention given to the nonlinearities that enter as thicknesses increase. The extrapolation shown in Ref. 25 appears to ignore this point entirely, while one of those shown in Ref. 33 ignores it as well. As a consequence, we believe that the extrapolated values of the asymmetries reported in these references are probably too low. As a final criticism of recent Mott polarization measurements, we address the claim made by Campbell et al. ${ }^{33}$ that by comparison with foil-thicknessextrapolation methods their technique of energy-loss extrapolation more successfully eliminates systematic errors in the value of the Sherman function $S$. While we believe that their studies may be the most precise ever performed, and while we generally concur with their claim, we believe that their published analysis ${ }^{78}$ relies too heavily upon a specious comparison of their relative data [normalized to Holwarth and Meister's theoretical value of $S$ at $100 \mathrm{keV}$ (Refs. 44 and 45)] with the absolute data of Mikaelyan et al. ${ }^{18}$ and Van Klinken ${ }^{20}$ obtained in Mott doublescattering experiments. Such double-scattering experiments are known ${ }^{24}$ to be susceptible to energy-related systematic effects that are produced by energy-dependent variations in beam profile and emittance and are quite independent of effects associated with foil-thickness extrapolations.

In connection with systematic effects related to the beam energy, $E$, we point out that since $S$ is a function of $E$ as well as $\theta$, an error in $E$ will produce an error in the calibration of the polarimeter. For the results we obtained with our conventional polarimeter, we believe that we successfully minimized such an error by referencing our beam energy to the known energies of ${ }^{109} \mathrm{Cd}$ internal conversion electrons rather than relying on voltage measurements obtained with standard high-voltage probes. While some users of Mott polarimeters are aware ${ }^{79}$ of the potential calibration problems introduced by the use of standard probes, ${ }^{80}$ we suspect that many others are not.

We conclude this paper with a few brief remarks about future studies. It seems to us that in light of the increasing application of polarized electrons to a variety of fields of physics, there is a strong, urgent need for the development of an electron polarimeter with absolute calibration. To this end, we suggest that precision Mott-doublescattering experiments be carefully carried out, that new theoretical computations of the Sherman function be performed using the latest atomic data bases, that compact polarimeter energy extrapolations and foil-thickness extrapolations be performed with the highest resolution possible, and that a standard polarimeter, free from Mott uncertainties, be developed. We believe that this last point is 
of the utmost importance and deserves the prompt attention of polarized electron researchers. Although an optical electron polarimeter ${ }^{81-83}$ may prove to be most suitable for the purpose of a standard, we suggest that other avenues $^{1,2}$ be explored as well. Without the development of a reliable calibration technique for polarimeters, we believe that the ultimate capabilities of polarized electron physics will never be fulfilled.

\section{ACKNOWLEDGMENTS}

We wish to thank Professor W. Raith and Professor G. Baum of Bielefeld for their major contributions to the design and construction of the polarimeter. We also wish to acknowledge the discussions we had with Professor
Raith regarding the data analysis and with Professor F. B. Dunning and Professor G. K. Walters of Rice and Dr. R. J. Celotta and Dr. D. T. Pierce of the National Bureau of Standards regarding the precision of the cylindrical and spherical Mott polarimeters. In addition, we wish to thank the Rice group for allowing us to reprint illustrations of these polarimeters. Finally, we wish to express our appreciation to Professor V. W. Hughes of Yale for providing encouragement for the pursuit of this research. We acknowledge with appreciation the financial support of the U.S. National Science Foundation under Grants No. PHY81-05035 (Yale) and No. PHY83-02868 (CCNY) and the City University of New York under Grant No. PSC-CUNY RF 664258. One of us (M.S.L.) received additional support from the Alfred P. Sloan Foundation.
*Present address: Department of Physics, University of Virginia, Charlottesville, VA 22901.

†Present address: Department of Physics, University of Missouri-Rolla, Rolla, MO 65401.

1J. Kessler, Polarized Electrons, 2nd ed. (Springer-Verlag, Berlin, 1985).

${ }^{2}$ R. J. Celotta and D. T. Pierce, Adv. At. Mol. Phys. 16, 102 (1980).

${ }^{3}$ G. Baum, in Polarization Phenomena in Nuclear Physics (Santa $\mathrm{Fe}, 1980$ ), Proceedings of the Fifth International Symposium on Polarization Phenomena in Nuclear Physics, AIP Conf. Proc. No. 69, edited by G. G. Ohlson, R. E. Brown, N. Jarmie, M. W. McNaughton, and G. M. Hale (AIP, New York, 1981), p. 785.

${ }^{4}$ M. S. Lubell, in Atomic Physics 5, edited by R. Marrus, M. Prior, and H. Shugart (Plenum, New York, 1977), p. 325.

${ }^{5}$ H. C. Siegmann, Phys. Rep. 17, 37 (1975).

${ }^{6} \mathrm{~W}$. Raith, in Atomic Physics, edited by B. Bederson, V. W. Cohen, and F. M. J. Pichanick (Plenum, New York, 1969), p. 389.

${ }^{7}$ V. H. Hughes, in Proceedings of the Fifth International Conference on High Energy Accelerators, edited by M. Grilli (Comitato Nazionale par l'Energia Nucleare, Rome, Italy, 1966), p. 531.

${ }^{8}$ D. T. Pierce, F. Meier, and P. Zürcher, Phys. Lett. 51A, 465 (1975); Appl. Phys. Lett. 26, 670 (1975); D. T. Pierce and F. Meier, Phys. Rev. B 13, 5484 (1976).

${ }^{9}$ See, for example, D. T. Pierce, R. J. Celotta, G.-C. Wang, W. N. Unertl, A. Galejs, C. E. Kuyatt, and S. R. Mielczarek, Rev. Sci. Instrum. 51, 478 (1980).

${ }^{10}$ C. S. Feigerle, D. T. Pierce, A. Seiler, and R. J. Celotta, Appl. Phys. Lett. 44, 866 (1984).

${ }^{11}$ H. J. Drouhin, C. Hermann, and G. Lampel, Phys. Rev. B 31, 3859 (1985); 31, 3872 (1985).

${ }_{12}$ N. F. Mott, Proc. R. Soc. London, Ser. A 124, 425 (1929); 135, 429 (1932); C. G. Shull, C. J. Chase, and F. E. Meyers, Phys. Rev. 63, 29 (1943).

${ }^{13}$ N. F. Mott and H. S. W. Massey, The Theory of Atomic Collisions, 3rd ed. (Oxford University Press, London, 1965), p. 263.

${ }^{14}$ H. A. Tolhoek, Rev. Mod. Phys. 28, 277 (1956).

${ }^{15} \mathrm{H}$. Frauenfelder and R. M. Steffen, in Alpha, Beta, and Gamma-Ray Spectroscopy, edited by K. Siegbahn (NorthHolland, Amsterdam, 1965), Vol. 2, p. 1431.
${ }^{16}$ J. S. Greenberg, D. P. Malone, R. L. Gluckstern, and V. W. Hughes, Phys. Rev. 120, 1393 (1960); D. M. Lazarus and J. S. Greenberg, Phys. Rev. D 2, 45 (1970).

${ }^{17}$ V. A. Apalin, I. Ye. Kutikov, J. J. Lukashevich, L. A. Mikaelyan, G. V. Smirnov, and P. Ye. Spivak, Nucl. Phys. 31, 657 (1962).

${ }^{18}$ L. Mikaelyan, A. Borovoi, and E. Denisov, Nucl. Phys. 47, 328 (1963).

${ }^{19}$ V. Eckardt, A. Ladage, and U. Moellendorff, Phys. Lett. 13, 53 (1964).

20J. Van Klinken, Nucl. Phys. 75, 161 (1966).

${ }^{21}$ W. Eckstein, Institut für Plasmaphysik, Garching bei München, Internal Report No. IPP 7/1, 1970 (unpublished).

${ }^{22}$ P. F. Wainwright, M. J. Alguard, G. Baum, and M. S. Lubell, Rev. Sci. Instrum. 49, 571 (1978).

${ }^{23}$ C. Y. Prescott et al., Phys. Lett. 77B, 347 (1978).

${ }^{24}$ M. J. Alguard, J. E. Clendenin, R. D. Ehrlich, V. W. Hughes, J. S. Ladish, M. S. Lubell, K. P. Schüler, G. Baum, W. Raith, and R. H. Miller, Nucl. Instrum. Meth. 163, 29 (1979).

${ }^{25}$ L. A. Hodge, T. J. Moravec, F. B. Dunning, and G. K. Walters, Rev. Sci. Instrum. 50, 5 (1979).

${ }^{26}$ B. Reihl, M. Erbudak, and D. M. Campbell, Phys. Rev. B 19, 6358 (1979); R. Raue, H. Hopster, and E. Kisker, Rev. Sci. Instrum. 55, 383 (1984).

${ }^{27}$ W. Wübker, R. Möllenkamp, and J. Kessler, Phys. Rev. Lett. 49, 272 (1982); G. F. Hanne and J. Kessler, J. Phys. B 9, 791 (1976); K. Jost and J. Kessler, Z. Phys. 195, 1 (1966).

${ }^{28}$ D. T. Pierce and R. J. Celotta, Adv. Electron. Electron Phys. 56, 219 (1981).

${ }^{29}$ G. E. Hanne, Phys. Rep. 95, 95 (1982).

${ }^{30}$ F. Schäfers, G. Schönhense, and U. Heinzmann, Z. Phys. A 304, 41 (1982); U. Heinzmann, J. Phys. B 11, 399 (1978).

${ }^{31}$ H. Riechert, S. F. Alvarado, A. N. Titkov, and V. I. Safarov, Phys. Rev. Lett. 52, 2297 (1984).

${ }^{32}$ L. G. Gray, M. W. Hart, F. B. Dunning, and G. K. Walter, Rev. Sci. Instrum. 55, 88 (1984).

${ }^{33}$ D. M. Campbell, C. Hermann, G. Lampel, and R. Owen, J. Phys. E 18, 663 (1985).

${ }^{34}$ G. D. Fletcher, M. J. Alguard, T. J. Gay, V. W. Hughes, P. F. Wainwright, M. S. Lubell, and W. Raith, Phys. Rev. A 31, 2854 (1985).

${ }^{35}$ G. Baum, M. Moede, W. Raith, J. Phys. B 18, 531 (1985); D. Hils, M. V. McCusker, H. Kleinpoppen, and S. J. Smith, Phys. Rev. Lett. 29, 398 (1972). 
${ }^{36}$ R. Allenspach, M. Taborelli, and M. Landolt, Phys. Rev. Lett. 55, 2599 (1985).

${ }^{37}$ H. Frauenfelder, R. Bobone, E. von Goeler, N. Levine, H. R. Lewis, R. N. Peacock, A. Rossi, and G. DePasquali, Phys. Rev. 106, 386 (1957).

${ }^{38}$ A. R. Brosi, A. I. Galonsky, B. H. Kettele, and H. B. Willard, Nucl. Phys. 33, 353 (1962).

${ }^{39}$ J. H. Bartlett and R. E. Wilson, Proc. Am. Acad. Arts Sci. 74, 53 (1940).

40J. A. Doggett and V. L. Spencer, Bull. Am. Phys. Soc. 1, 37 (1956).

${ }^{41}$ Y. N. Yadev, Proc. Phys. Soc. London, Sect. A 68, 348 (1955).

${ }^{42}$ R. M. Curr, Proc. Phys. Soc. London, Sect. A 68, 156 (1955).

${ }^{43}$ H. Feshbach, Phys. Rev. 88, 295 (1951).

${ }^{44} \mathrm{G}$. Holzwarth and H. J. Meister, Nucl. Phys. 59, 56 (1964).

${ }^{45}$ Tables of Asymmetry, Cross-Section and Related Functions for Mott Scattering of Electrons by Screened Gold and Mercury Nuclei, edited by G. Holzwarth and H. J. Meister (Institut für Theoretische Physik der Universität München, Germany, 1964).

46Shin-R Lin, Phys. Rev. A 133, 965 (1964).

${ }^{47}$ W. Bühring, Z. Phys. 212, 61 (1968).

${ }^{48}$ N. Sherman, Phys. Rev. 103, 1601 (1956).

${ }^{49}$ N. Sherman and D. F. Nelson, Phys. Rev. 114, 1541 (1959).

${ }^{50}$ M. J. Alguard, V. W. Hughes, M. S. Lubell, and P. F. Wainwright, Phys. Rev. Lett. 39, 334 (1977).

${ }^{51}$ M. S. Lubell, in Coherence and Correlation in Atomic Collisions, edited by H. Kleinpoppen and J. F. Williams (Plenum, New York, 1980), p. 663.

${ }^{52}$ G. D. Fletcher, M. J. Alguard, T. J. Gay, V. W. Hughes, C. W. Tu, P. F. Wainwright, M. S. Lubell, W. Raith, and F. C. Tang, Phys. Rev. Lett. 48, 1671 (1982).

${ }^{53}$ T. J. Gay, G. D. Fletcher, M. J. Alguard, V. W. Hughes, P. F. Wainwright, and M. S. Lubell, Phys. Rev. A 26, 3664 (1982).

${ }^{54} \mathrm{H}$. Frauenfelder and A. Rossi, in Methods of Experimental Physics, edited by L. C. L. Yuan and C. S. Wu (Academic, New York, 1963), Vol. 5, Part B, p. 214.

${ }^{55}$ G. Moliere, Z. Naturforsch. 2a, 133 (1947); 3a, 78 (1948).

${ }^{56} \mathrm{~B}$. Mühlschlegel and H. Koppe, Z. Phys. 150, 474 (1958).

${ }^{57}$ H. Wegener, Z. Phys. 151, 252 (1958).

${ }^{58} \mathrm{~L}$. Braicovich and B. de Michelis, Nuovo Cimento 58B, 269 (1968).

${ }^{59}$ H. Boersch, R. Schliepe, and K. E. Schriefl, Nucl. Phys. A163, 625 (1971).
${ }^{60}$ V. Hnizdo, Nucl. Instrum. Meth. 109, 503 (1973).

${ }^{61}$ V. W. Hughes, R. L. Long, Jr., M. S. Lubell, M. Posner, and W. Raith, Phys. Rev. A 5, 195 (1972).

${ }^{62}$ B. Planskoy, Nucl. Instrum. Meth. 61, 285 (1968).

${ }^{63}$ G. W. Ford and C. J. Mullin, Phys. Rev. 110, 520 (1958).

${ }^{64}$ R. E. Burge and G. H. Smith, Proc. Phys. Soc. London 76, 673 (1962)

${ }^{65}$ T. A. Weber, R. T. Deck, and C. J. Mullin, Phys. Rev. 130, 660 (1963).

${ }^{66}$ G. Missoni, C. E. Dick, R. C. Placious, and J. W. Motz, Phys. Rev. A 2, 2309 (1970).

${ }^{67}$ D. H. Rester and N. Edmonson, Phys. Rev. A 4, 147 (1971).

${ }^{68}$ M. Inokuti, Rev. Mod. Phys. 43, 297 (1971).

${ }^{69}$ J. W. Cooper and H. Kolbenstvedt, Phys. Rev. A 5, 677 (1972).

${ }^{70}$ J. N. Das and A. N. Konar, Nuovo Cimento 21A, 289 (1974).

${ }^{71}$ H. Schmoranzer, H. Grabe, and B. Schiewe, Appl. Phys. Lett. 26, 483 (1975).

72B. D. Grachev, S. S. Kozlovskii, Yu. S. Korobochko, V. I. Mineev, and A. F. Petrochenko, Zh. Eksp. Teor. Fiz. 79, 1641 (1980) [Sov. Phys.-JETP 52, 827 (1980)].

${ }^{73}$ Note that the value of $\alpha$ given on p. 211 of Ref. 61 should read $0.0025 \mathrm{~cm}^{2} / \mu \mathrm{g}$ instead of $0.0035 \mathrm{~cm}^{2} / \mu \mathrm{g}$.

${ }^{74} \mathrm{R}$. J. Celotta, private communication.

${ }^{75}$ See, for example, E. Segre, Nuclei and Particles, 2nd ed. (Benjamin, Reading, Massachusetts, 1977), p. 28-41.

${ }^{76}$ C. Møller, Ann. Physik 14, 568 (1932).

${ }^{77}$ S. F. Alvarado, H. Riechert, and N. Christensen, Phys. Rev. Lett. 55, 2716 (1985).

${ }^{78} \mathrm{G}$. Lampel has informed us privately that an unpublished analysis of their data, which does not involve comparisons with other experiments but rather involves comparisons of slopes of their own extrapolations, leads to the same stated claim.

${ }^{79}$ F. B. Dunning and G. K. Walters, private communication.

${ }^{80}$ Standard high-voltage probes use high-resistance voltage dividers that are calibrated under low-humidity conditions. While the probe-housing material is usually chosen to resist absorption of water vapor, degradation does occur with time, with the consequence that changes in the surface resistivity eventually affect the accuracy of the calibration.

${ }^{81}$ P. S. Farago and J. S. Wykes, J. Phys. B 2, 747 (1969).

${ }^{82}$ M. Eminyan and G. Lampel, Phys. Rev. Lett. 45, 1171 (1980).

${ }^{83}$ T. J. Gay, J. Phys. B 16, L553 (1983). 

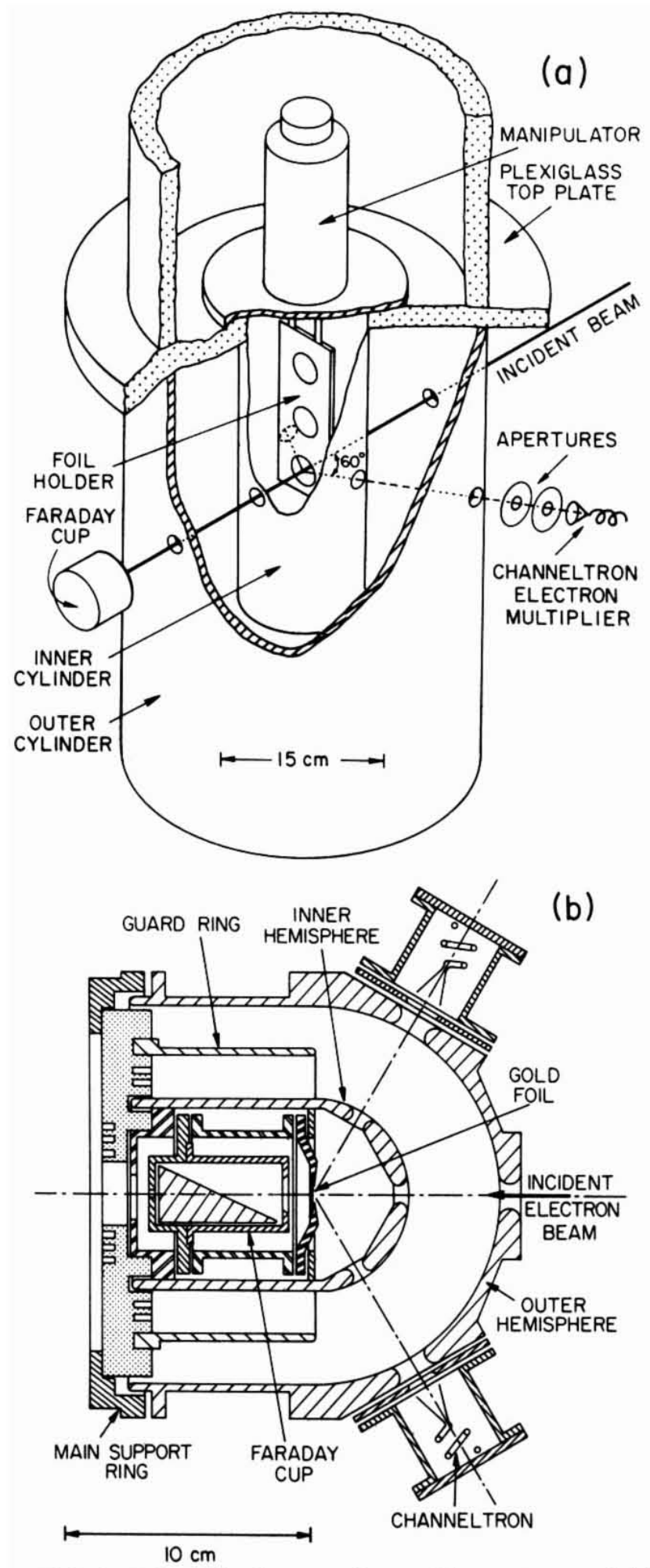

FIG. 3. Schematic diagram of essential components of (a) cylindrical Mott polarimeter taken from Ref. 25 and (b) spherical Mott polarimeter taken from Ref. 32. 